\title{
The Effect of Deviation Due to the Manufacturing Accuracy in the Parameters of an MPP on Its Acoustic Properties: Trial Production of MPPs of Different Hole Shapes Using 3D Printing
}

\author{
Kimihiro Sakagami ${ }^{1, *(D)}$, Midori Kusaka ${ }^{1}$, Takeshi Okuzono ${ }^{1}$ (D) and Shinsuke Nakanishi ${ }^{2}$ (D) \\ 1 Environmental Acoustics Laboratory, Department of Architecture, Graduate School of Engineering, \\ Kobe University, Rokko, Nada, Kobe 657-8501, Japan; kusakamidori6@gmail.com (M.K.); \\ okuzono@port.kobe-u.ac.jp (T.O.) \\ 2 Department of Architectural Technology, Faculty of Engineering, Hiroshima Institute of Technology, \\ Hirokoshingai 5-1-1, Kure, Hiroshima 737-0112, Japan; s.nakanishi.9a@it-hiroshima.ac.jp \\ * Correspondence: saka@kobe-u.ac.jp
}

Received: 4 August 2020; Accepted: 12 August 2020; Published: 14 August 2020

\begin{abstract}
In this study, we discuss the effect of the manufacturing accuracy of a microperforated panel (MPP) produced by 3D printers on acoustic properties through measured and calculated results as a pilot study. The manufacturing costs of MPPs have long been one of their shortcomings; however, with recent developments in the manufacturing process, low-cost MPPs are now available. In a further attempt at reducing the cost, 3D printing techniques have recently been considered. Cases of trial production of MPPs manufactured by 3D printing have been reported. When introducing such new techniques, despite the conventional microdrill procedure, manufacturing accuracy can often become an issue. However, there are few studies reporting the effect of manufacturing accuracy on the acoustic properties in the case of 3D-printed MPPs. Considering this situation, in this pilot study, we attempted to produce MPPs with circular and rectangular perforations using a consumer 3D printer of the additive manufacturing type. The hole sizes of the specimens were measured, and the accuracy was evaluated. The normal incidence absorption coefficient and specific impedance were measured using an impedance tube. The measured results were compared with the theoretical values using Guo's model. Through these basic studies, the MPPs produced by an additive manufacturing 3D printer demonstrated good sound absorption performance; however, due to the large deviations of parameters, the agreement with the theoretical values was not good, which suggests that it is difficult to predict the acoustic properties of MPPs made by a consumer-grade additive manufacturing 3D printer.
\end{abstract}

Keywords: microperforated panel; 3D printer; additive manufacturing; sound absorption; manufacturing accuracy; theoretical prediction

\section{Introduction}

\subsection{Background}

Microperforated panels (MPPs) absorb sound energy, in principle, by the same mechanism as of a conventional perforated panel, which forms a Helmholtz resonator with the holes and the air-back cavity. However, MPPs have much smaller parameters, i.e., thickness and hole diameter are smaller than $1 \mathrm{~mm}$ and perforation ratio is less than $1 \%$, so that acoustic resistance and reactance suitable for better sound absorption performance than conventional perforated panels is realized. 
In the pioneering work on MPPs [1-3], Maa proposed MPPs and established a predicting theory for designing an optimized sound absorber using MPPs. Later, Fuchs et al. and other researchers made various implementation experiments for practical uses, which contributed to draw attention to this promising alternative for a next generation sound absorber that inherits recyclability and sustainability [4-6]. Although, in Japan, there are only a few commercial products available, a considerable number of products are commercially available in Europe, the US, and other countries. Among them, there are many products of superior design and additional aesthetic value [7].

Regarding the spread of this attractive sound absorbing material, the largest problem is the high-precision manufacturing technique and its cost. In contrast to the "First generation MPP", which mainly uses metal sheets, recently, the "Second generation MPP" uses softer materials, such as, plastics, resins, etc. With the recent developments in manufacturing techniques, the cost problem is reduced [8-10]. There are various studies that have been performed on MPPs produced by 3D printing, which is one of the alternative methods to produce MPPs [11,12]. In addition, the recent development of this emerging technique has been applied to more complex metamaterial panels by employing its flexible producibility, which can now be considered as it goes into a next stage.

\subsection{Sound Absorption Performance of MPPs and Manufacturing Accuracy}

In the case of producing MPPs using a 3D printer, the manufacturing accuracy of the hole diameters of specimens and its effect on the acoustic properties are easily assumed. However, there are few publications that discuss the method to produce MPPs with a 3D printer and the accuracy of printed specimens. For example, Liu et al. [11] used a stereolithography (SLA) type 3D printer to produce an MPP, and its measured absorption characteristics were in good agreement with the calculated results from Maa's theory. However, they only mentioned that the resolution of the layer was approximately $0.025 \mathrm{~mm}$, which was sufficient, and did not provide any more information to be reproducible. According to the description in the paper, their specimens had a relatively large hole diameter, which may have helped to maintain accuracy. They mentioned that the thickness had a deviation of $2 \%$.

There are some other examples of trial productions of sound absorbers using 3D printers; however, most do not provide detailed information regarding the production. A perforated panel with a larger diameter and slanted holes was studied by Carbajo et al. [13]. Their samples showed good agreement with the theoretical results; however, the method of production and manufacturing accuracy of the specimens were not clearly mentioned. On the other hand, Nakanishi [14] studied a resonance type absorber with additional backing structures by using an additive manufacturing 3D printer. In his study, the structures were not composed of parts requiring a high accuracy; instead, various examples of absorbers made by additive manufacturing 3D printer were presented.

Boulvert et al. [12] made trial productions of sound absorbers called micro-lattices by using an additive manufacturing 3D printer. They attempted to study the manufacturing accuracy and prediction of its absorption characteristics by using the Johnson-Champoux-Allard-Lafarge (JCAL) model. In this work, the accuracy was affected by the cross-sectional shape of the nozzle, which was approximately $8 \%$ and though they stated that, while not measurable, there were deviations attributed to heat shrinking and microgrooves in the nozzle.

Although it is not related to 3D printing, Pan and Martellotta [15] performed trial productions of MPP with films of $5.0 \mathrm{~mm}$ thickness with microdrills. The hole diameter of their specimens showed a Gaussian distribution from 0.2 to $0.35 \mathrm{~mm}$ around the targeted value of $0.3 \mathrm{~mm}$.

Therefore, they calculated the "effective hole diameter" from the measured values of the area of the holes from their diameters, and they successfully predicted the absorption characteristics with Maa's theory using these "effective hole diameters".

Considering these results, it is necessary to clarify the effect of the manufacturing accuracy on the absorption characteristics of an MPP in any method of production, in particular when 3D printing is used. Therefore, in this study, as a pilot study, we produced MPPs with different holes by an additive 
manufacturing 3D printer and studied how the hole shape and size deviated from the target values, and how they affected the predictability with the existing theory.

\subsection{Purpose of the Present Study}

As a basic study on the present issue, trial productions of MPPs with different shapes of perforations were made using an additive manufacturing $3 \mathrm{D}$ printer. The absorption characteristics of the specimens produced by the printer were measured using an impedance tube. The measured values were compared with the theoretical values to evaluate their agreement, and the effect of the manufacturing accuracies of the specimens on the acoustical properties of the specimens were discussed.

As for the hole shape of an MPP, the majority of the previous studies discussed circular holes only, except for a micro-slit. The reason why can be interpreted as the manufacturing cost: circular holes are easier to produce than other shapes, such as rectangular, triangular, etc. In the theoretical sense, polygonal shapes cause higher acoustic resistance due to the larger hydraulic radius [16]; thus, an experimental trial would be of interest. If those more complex shape holes can be easily produced by the 3D printing method, this will add variety in the design alternatives. Therefore, in this study, 3D printed MPPs with square shaped holes were also included.

\section{Preparation of the Specimens}

In this study, an additive manufacturing 3D printer was used for the trial production of MPP specimens, and their acoustic properties were measured by experiment in an impedance tube. We also discussed the theoretical predictions. In general, with respect to the manufacturing accuracy, stereolithography (SLA) is well known to be more functional than additive manufacturing. However, in this study, considering cost and availability, a high-end consumer model additive manufacturing 3D printer (Maker Bot, Replicator 2) was used. The filaments were PLA (polylactic acid resin).

We designed the three specimens with the parameters shown in Table 1. Each specimen was preliminarily considered by theoretical calculations using Guo's model [16], and those parameters were decided by discussing the calculated results. Two of the three were designed to have square holes, which were considered difficult to produce by the conventional manufacturing method. The other was designed to have a circular hole. The sizes of the holes of the specimens were decided to be larger than typical MPPs in consideration of reproducibility. However, the sizes of the holes and the thicknesses of the manufactured specimens showed deviations. Therefore, the actual sizes of all holes in all specimens were measured by a measuring microscope (J\&T, JT-03-095) and the thickness by a digital thickness gauge (J\&T, JT-49-012). The measured values of the parameters are shown in Table 2.

Table 1. Targeted parameters for trial production using a 3D printer.

\begin{tabular}{cccccc}
\hline Specimen & Hole Shape & Side Length $(\mathbf{m m})$ & Radius $(\mathbf{m m})$ & Thickness $(\mathbf{m m})$ & Perforation Ratio $(\%)$ \\
\hline No.1 & Square & 0.5 & - & 0.5 & 0.25 \\
No.2 & Square & 1.0 & - & 0.5 & 1.0 \\
No.3 & Circular & - & 0.5 & 0.5 & 0.785 \\
\hline
\end{tabular}

Table 2. Measured parameters of the resulting specimens made by 3D printer.

\begin{tabular}{cccccc}
\hline Specimen & Hole Shape & Side Length $(\mathbf{m m})$ & Radius $(\mathbf{m m})$ & Thickness $(\mathbf{m m})$ & Perforation Ratio $(\%)$ \\
\hline No.1 & Square & 0.767 & - & 0.608 & 0.587 \\
No.2 & Square & 0.883 & - & 0.644 & 0.781 \\
No.3 & Circular & - & 0.511 & 0.645 & 0.823 \\
\hline
\end{tabular}

From Table 2, in all specimens, the averaged measured hole sizes and thicknesses were different from the designed values in Table 1, which proves that there were manufacturing errors. These are considered to be the errors that occur in the process of hardening the hot-melt filament [12]. Regarding the hole shape (as shown in Figure 1, which is an example of the magnified photograph of a part of 
a specimen), in the case of square holes, the edges are rounded and not precisely square. As for the thickness, the errors were caused by the same reason: in the case of thickness, the horizontal precision of the workbench at the time of production was affected. The error in the thickness was different from position to position in a specimen. These deviations can also be attributed to the following circumstances: (1) in the design stage, a "try-and-error" method is often employed to adjust the dimensions. For example, the resulting samples are not necessarily the same size as designed in the CAD data used. In such cases, we need to adjust the size defined in the CAD data; (2) shrinkage and expansion occur in the production, which depends on the temperature on each place of the sample surface; (3) it is quite difficult to obtain an accurate horizontal plane with the platform on which a sample is output-melt filament can flow by inclined platform which can cause an error; and (4) to obtain the defined thickness, the manufacturing is made by adding layers, but when a new layer is produced on an existing layer, the new layer which is hot can melt the existing layer again, leading to an error in its size. Due to these circumstances, the errors can appear rather randomly-the parameters of the sample may be smaller or larger than the targeted values.

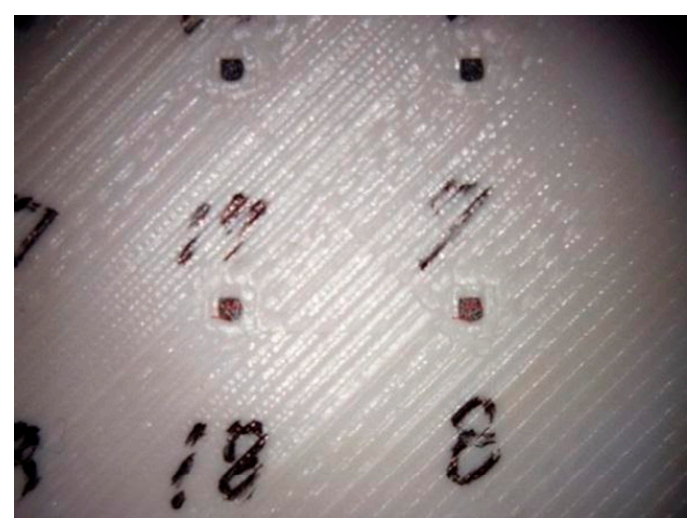

(a)

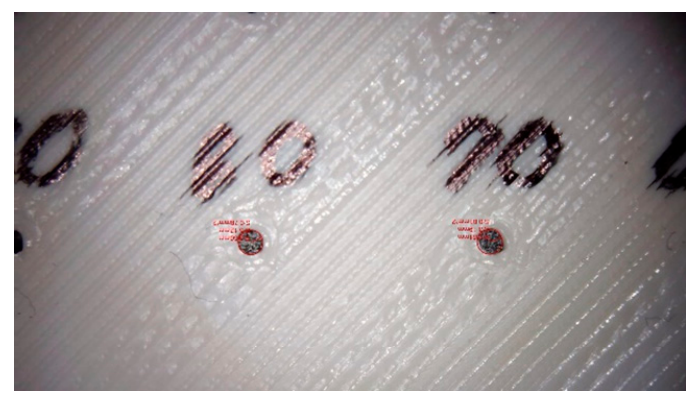

(b)

Figure 1. Examples of the magnified photograph of a portion of (a) a square hole sample (Specimen \#2) and (b) a circular hole sample (Specimen \#3).

Regarding the time required for production, in this study, the sample was $12 \mathrm{~cm}$ square, and made by adding five layers, which required 30-60 min for completing one sample. The accuracy may also depend on the production time, but it depends on the printer used.

The sizes of all 100 holes in each sample were measured using the measuring microscope. Regarding the specimens Nos. 1 and 2 (square hole), both the lengths of each hole were measured. For specimen No. 3 (circular hole), the average diameter is shown. The cumulative frequency distribution of all measured size data is shown in Figures $2-4$. 

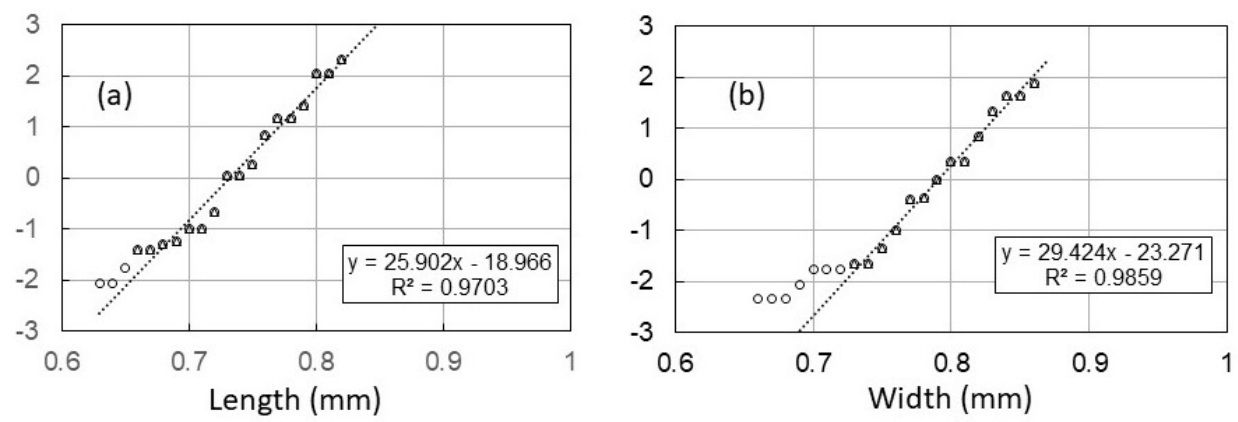

Figure 2. Normalized cumulative frequency distribution ( $\mathrm{z}$ value on the vertical axis) of the hole size of Specimen \#1. Sizes in the (a) vertical and (b) horizontal directions of the square holes.
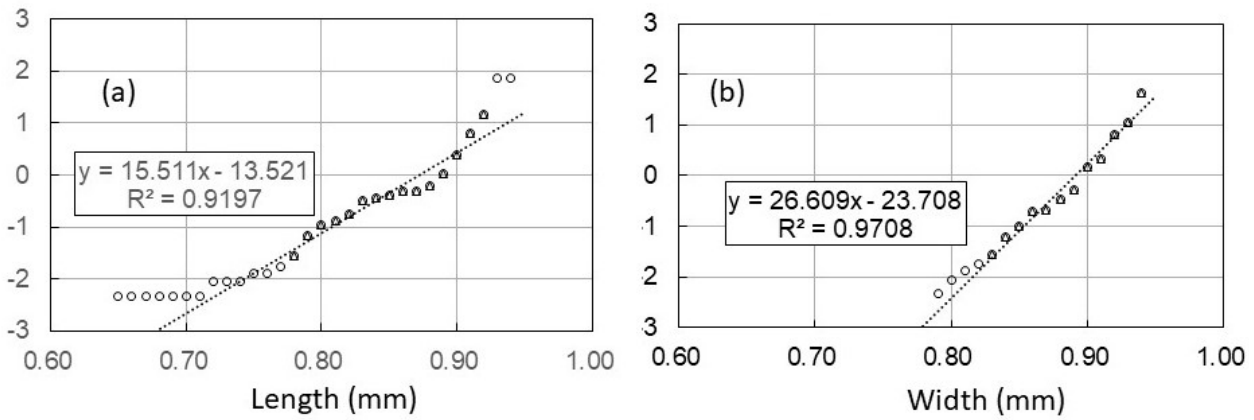

Figure 3. Normalized cumulative frequency distribution ( $z$ value on the vertical axis) of the hole size of Specimen \#2. Sizes in the (a) vertical and (b) horizontal directions of the square holes.

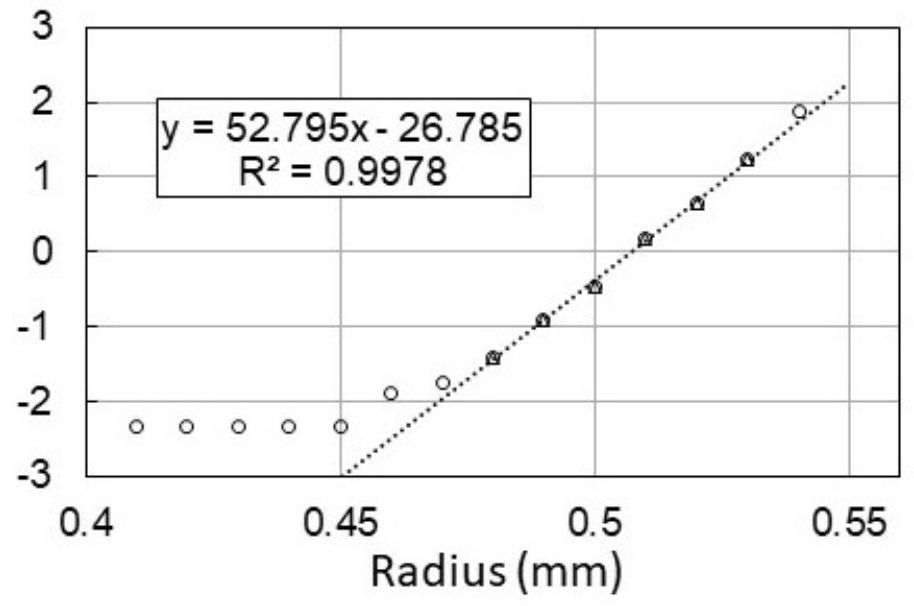

Figure 4. Normalized cumulative frequency distribution ( $\mathrm{z}$ value on the vertical axis) of the hole radius of Specimen \#3.

There was no systematic trend found in these distributions; therefore, these hole sizes were considered to be Gaussian distributions. This also means that the hole sizes were randomly distributed within a certain range, and therefore, that the errors were also random. Considering these results, we used the averaged values for the following theoretical calculations as input parameters.

\section{Measurement in an Impedance Tube and Theoretical Prediction}

\subsection{Outline of Measurement Method}

The measurements were performed using an impedance tube with a $10 \mathrm{~cm}$ square cross-section made of acrylic (Figure 5) and in accordance with JIS A 1405-2 (transfer function method. ISO 382 compatible). The depth of the air-back cavity was 25 and $50 \mathrm{~mm}$. The impedance tube was made 
in-house, and there was an error due to the residual absorption inside the tube; however, a correction was made using the theoretical calculation. In this system, we confirmed that it obtained the measured value for an aluminum MPP manufactured by precision processing to almost perfectly agree with the theoretical value.

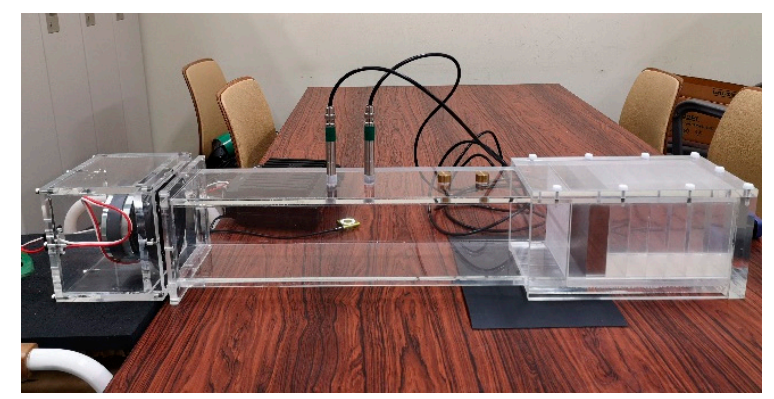

Figure 5. Experimental setup with an impedance tube.

\subsection{Prediction by Guo's Model}

In this study, we employed Guo's model, which is applicable to the cases with non-circular holes [16], and the calculated values were compared with the measured values. This theory is also introduced and reviewed in Bolton and Kim [17] and Herdtle et al. [18]. However, as the main part of the theory is concisely described in [19], one can consult this literature. Guo's model is a generalized form of classical Maa's theory, including the surface resistance. Therefore, it is essentially the same as Maa's theory, as they correspond to each other with a certain parameter. As for a circular hole, Guo's model has almost the same accuracy as of Maa's theory, which we have checked experimentally. Regarding the square holes, there has not been enough validation with experimental results, but Okuzono et al. [19] confirmed that it shows a good agreement with detailed FEM simulation results. In that, we decided to use this theory for the present purpose. Below, we summarize the theory of Guo's model according to [19].

Consider the surface impedance of a single-layer MPP absorber with an air-back cavity and a rigid wall, $Z_{\text {surf }}$; this is expressed with the transmission impedance of the MPP, $Z_{t}$, and the backing impedance of the rigid-backed air-layer, as:

$$
Z_{\text {surf }}=Z_{t}-j \rho_{0} c_{0} \cot \left(k_{0} L\right)
$$

where, $j, \rho_{0}, c_{0}, k_{0}$, and $L$ are the imaginary unit, air density $\left(1.205\left(\mathrm{~kg} / \mathrm{m}^{3}\right)\right)$, sound speed in air $(\mathrm{m} / \mathrm{s})$, wavenumber in air, and the depth of the air-back cavity $(\mathrm{m})$, respectively.

Now, $Z_{t}$ is expressed as:

$$
Z_{t}=\frac{j \omega \rho_{e} t}{\varepsilon}+\frac{\alpha 2 R_{s}}{\varepsilon}+\frac{j \omega \rho_{0} \delta}{\varepsilon}
$$

where, $\omega, t, \varepsilon$, and $\delta$ are the angular frequency ( $\mathrm{rad} / \mathrm{s})$, panel thickness (m), perforation ratio (-), and end correction factor, respectively.

The first term of Equation (2) represents the effect of viscous energy loss inside the hole, and here, $\rho_{e}$ is the effective density, which is expressed as the following in the case of a circular hole:

$$
\rho_{e}=\rho_{0} /\left[1-\frac{2}{\sqrt{-j}} \frac{J_{1}(s \sqrt{-j})}{J_{0}(s \sqrt{-j})}\right]
$$

where $J_{0}$ and $J_{1}$ are Bessel functions of the first kind of zero-th and first orders, respectively. The parameter $s$ is defined in the case of a circular hole:

$$
s=r \sqrt{\omega \rho_{0} / \eta}
$$


where $r$ is the radius (m) and $\eta$ is the viscosity of the air $(\mathrm{kg} / \mathrm{m} / \mathrm{s})$. In the case of a micro-perforation of an arbitrarily shaped cross-section, $\rho_{e}$ is given as:

$$
\rho_{e}=\rho_{0}\left(1+\frac{\sigma \varphi}{j \omega \rho_{0}} G_{c}(s)\right)
$$

where $\sigma$ is the flow resistivity $\left(\mathrm{Pa} \mathrm{s} / \mathrm{m}^{2}\right)$, which becomes $\sigma \varphi=7 \eta / r_{p}{ }^{2}$ in the case of a square hole, with $r_{p}$ as the parameter defined with the perimeter of the cell $l(\mathrm{~m})$ and the cross sectional area $S\left(\mathrm{~m}^{2}\right)$, which is the equivalent radius $r_{p}=2 S / l(\mathrm{~m}) . G_{c}(s)$ is given as:

$$
G_{c}(s)=-\frac{s}{4} \sqrt{-j} \frac{J_{1}(s \sqrt{-j})}{J_{0}(s \sqrt{-j})} /\left[1-\frac{2}{s \sqrt{-j}} \frac{J_{1}(s \sqrt{-j})}{J_{0}(s \sqrt{-j})}\right]
$$

with

$$
s=c \sqrt{8 \omega \rho_{0} / \sigma \phi}
$$

The second term in Equation (2) represents the end correction due to the surface admittance, and the parameter $R_{S}$ is given by the following equation:

$$
R_{S}=\sqrt{2 \eta \rho_{0} \omega} / 2
$$

The coefficient $\alpha$ in the second term of Equation (2) is dependent on the shape of the edge of the perforation, which means that Guo's model depends on the sharpness of the edge: when the cross-section of the hole edge is rounded, it is 2.0. If it is sharp, the value is 4.0. The third term is the end correction by the factor $\delta$, which is as follows:

For a circular hole, $\delta=\delta_{c}$ is [20]:

$$
\delta_{c}=0.85 d\left(1-1.13 \varepsilon^{1 / 2}-0.09 \varepsilon+0.27 \varepsilon^{3 / 2}\right)
$$

For a square hole, $\delta=\delta_{s}$ is:

$$
\delta_{s}=0.85 d\left(1-1.33 \varepsilon^{1 / 2}-0.07 \varepsilon+0.40 \varepsilon^{3 / 2}\right)
$$

Note that, when $\alpha=2$ and $\delta=0.85$, the Guo theory coincides Maa's theory in 1987 [2].

From the above formulae, once $Z_{\text {surf }}$ is obtained, the absorption coefficient is easily derived with the well-known conventional formula.

\section{Results and Discussion}

\subsection{Specimen No. 1}

Figure 6 shows the measured results (the normal incidence absorption coefficient and normalized specific acoustic impedance) in comparison with the calculated results from Guo's model. Regarding the absorption coefficient, the measured value shows its peak around $900 \mathrm{~Hz}$, but the theoretical value has a peak at lower frequencies, which is one of the main discrepancies. However, for the peak value, both values are near each other. The peak value of the measured absorption coefficient is rather high (0.95 ca.), which is comparable to that obtained by well-designed conventional MPP absorber. This fact proves that reasonably high peak absorption can be obtained by MPPs made by an additive manufacturing 3D printer, and they can be possibly used in practical purposes. However, considering the predictability of the existing theory, as the agreement between the measured and predicted value show large discrepancies, it is necessary to develop a better predicting theory or method. 

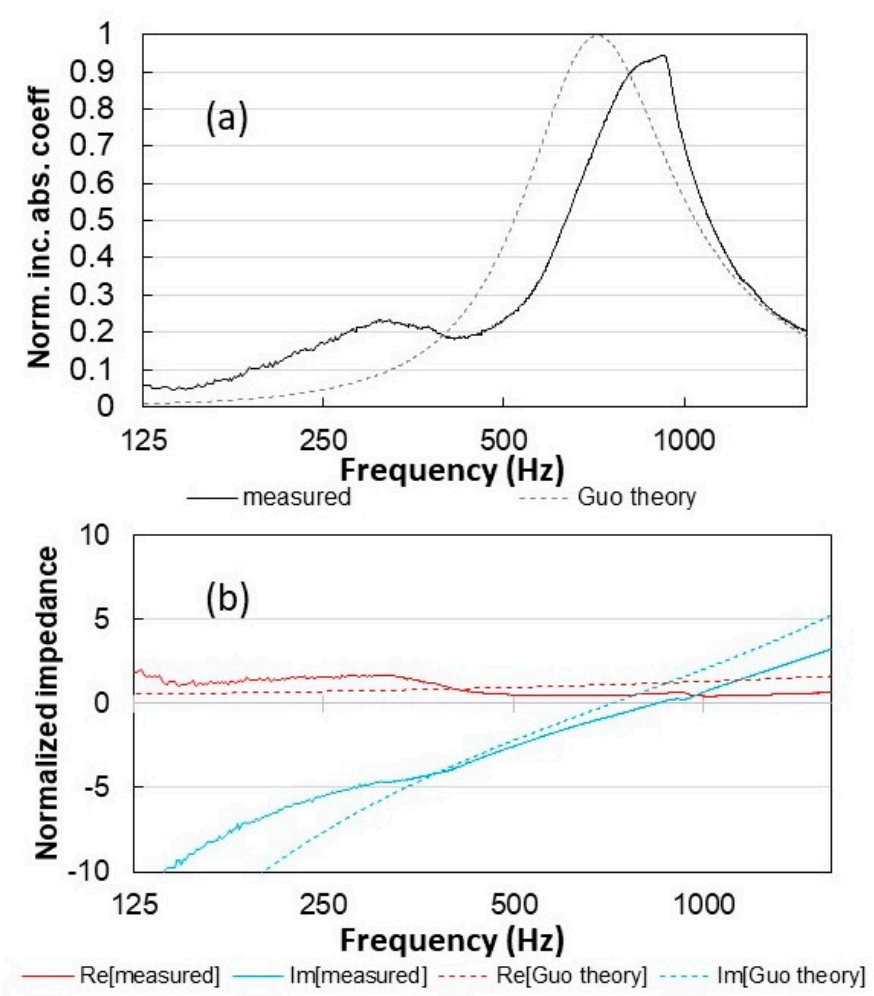

Figure 6. Comparison of the measured (solid line) and calculated (dashed line) values of the normal absorption coefficient (a) and specific impedance (b) of Specimen \#1, with an air-back cavity of $25 \mathrm{~mm}$ depth.

On the other hand, regarding the measured acoustic impedances, both the real and imaginary parts do not agree well with the theory. In particular, the discrepancy of the zero-crossing frequencies of the imaginary parts is directly reflected in the discrepancy in the peak frequencies. The main cause of this discrepancy is considered to be the deviation of the hole sizes and related parameters and the inhomogeneity resulting from this. The difference in the perforation ratio in each part of the specimen is inferred to have a dominant effect on both the peak value and frequency.

For the case of the cavity depth of $50 \mathrm{~mm}$, the results are shown in Figure 7. In this case, the peak frequency shifts to lower frequencies than that of the $25 \mathrm{~mm}$ case, which is as known as the general behavior of MPPs. The peak value was lower than in the $25 \mathrm{~mm}$ case. The specific impedance of the MPP specimen itself is the same; however, with the change in the depths of the air-back cavity, the total impedance, i.e., the surface impedance of the absorber, changes. Considering that the peak appears at the frequency where the imaginary part of the total impedance becomes zero, the peak value is determined by the real part (acoustic resistance) of the absorber at the frequency. Therefore, the behavior of the peak value and frequency cannot be in a simple relationship. However, the facts that are observed from the graphs are qualitatively the same as the discussion for the $25 \mathrm{~mm}$ case: the peak frequency and value do not agree well with the values predicted by Guo's model. In this case, the peak value is lower than the $25 \mathrm{~mm}$ case, however, it is still around 0.7, which can be reasonably high and effectively used in some practical purposes. As similar discussions were obtained both in the cases of 25 and $50 \mathrm{~mm}$ for the other specimens, only the results for the $25 \mathrm{~mm}$ case are demonstrated in the following. 

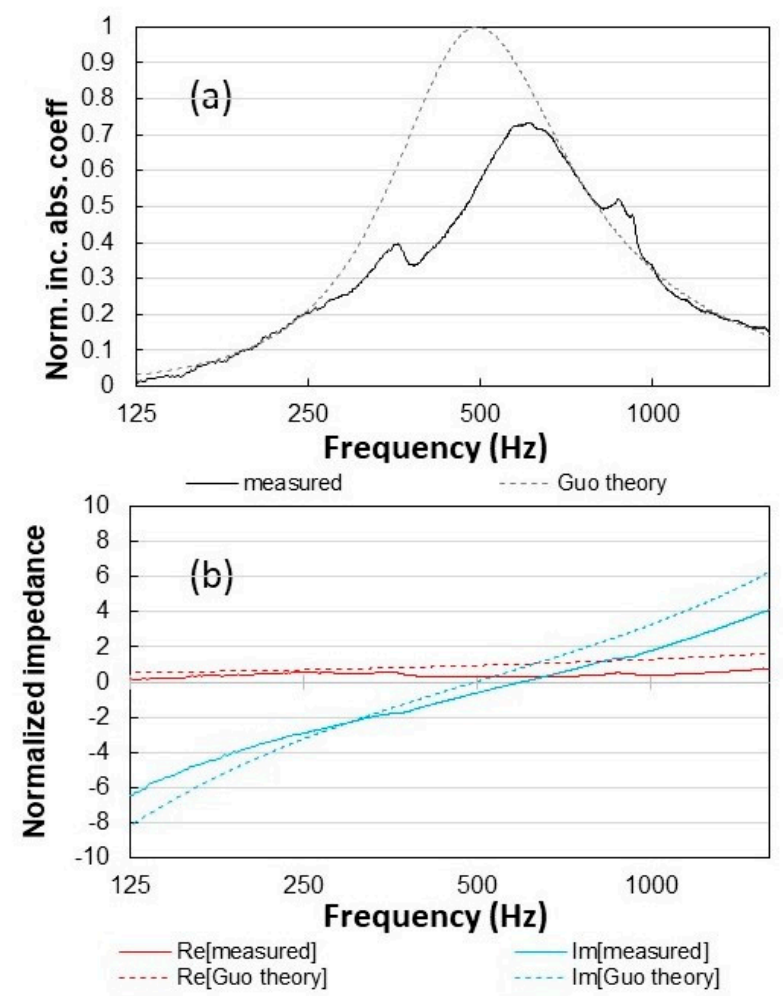

Figure 7. Comparison of the measured (solid line) and calculated (dashed line) values of the normal absorption coefficient (a) and specific impedance (b) of Specimen \#1, with an air-back cavity of $50 \mathrm{~mm}$ depth.

\subsection{Specimen No. 2}

Figure 8 shows the measured and theoretical results for specimen No. 2 (cavity depth: $25 \mathrm{~mm}$ ). As for the normal absorption coefficient, there is a clear peak with the peak value larger than 0.8 at $1 \mathrm{kHz}$ ca. However, the peak frequency is shifted to lower frequencies in the theory. The theoretical peak value is somewhat larger than the measured one. Regarding the impedance, again the same discussion is obtained as in the case of specimen No. 1.

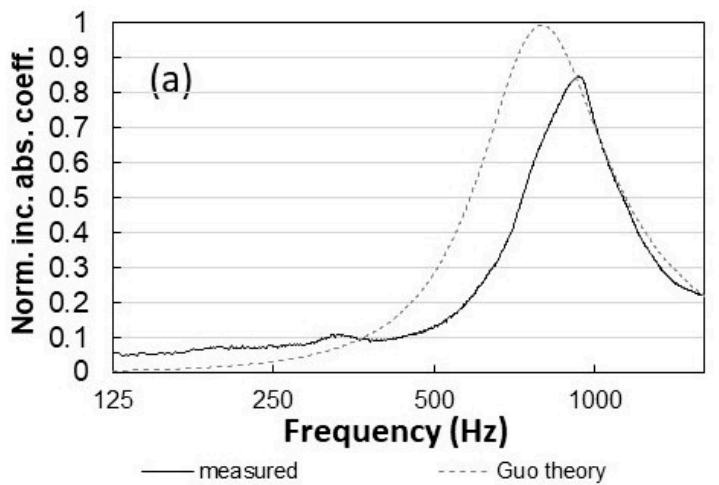

Figure 8. Cont. 


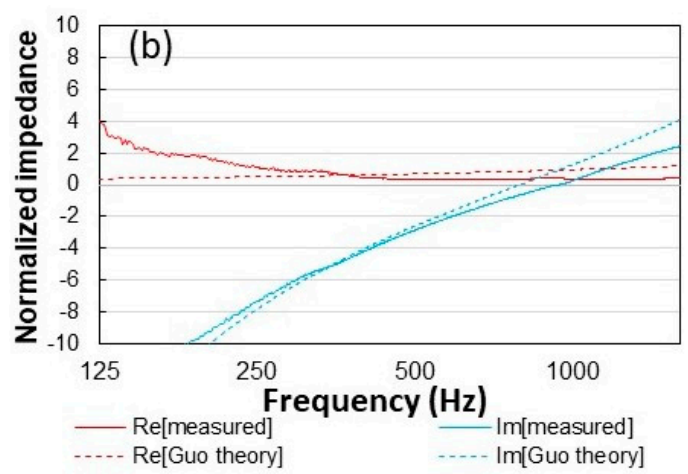

Figure 8. Comparison of the measured (solid line) and calculated (dashed line) values of the normal absorption coefficient (a) and specific impedance (b) of Specimen \#2, with an air-back cavity of $25 \mathrm{~mm}$ depth.

\subsection{Specimen No. 3}

Figure 9 shows the measured and theoretical results for No. 3 (cavity depth: $25 \mathrm{~mm}$ ). Although the peak frequencies of the measured and theoretical values were not in agreement, the peak values were in good agreement. The qualitative features of the graphs were somewhat in agreement when compared with the other two.
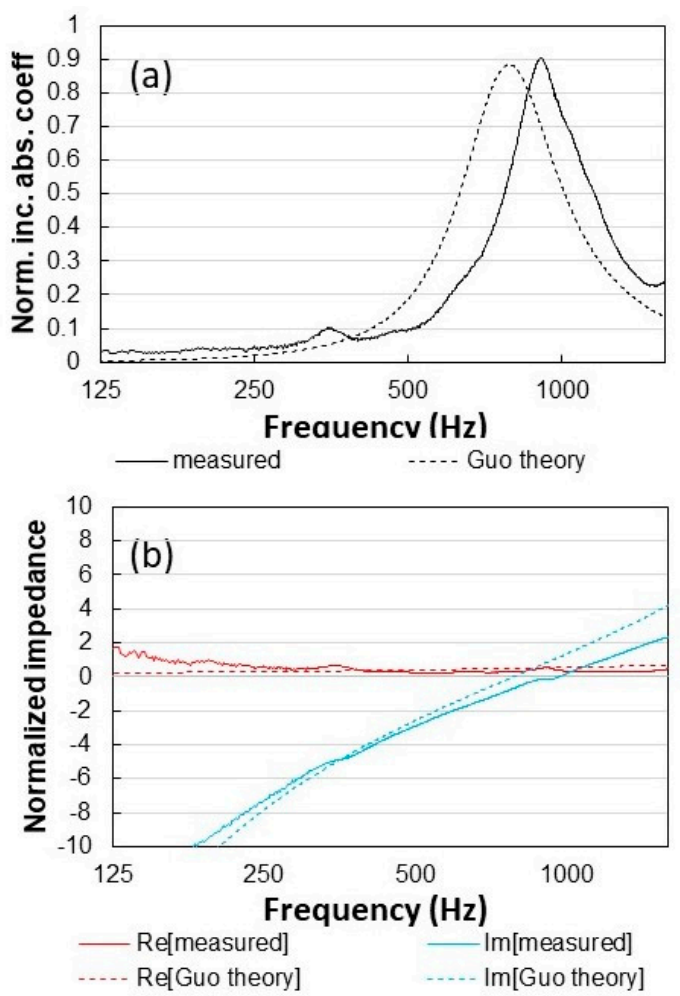

Figure 9. Comparison of the measured (solid line) and calculated (dashed line) values of the normal absorption coefficient (a) and specific impedance (b) of Specimen \#3, with an air-back cavity of $25 \mathrm{~mm}$ depth.

Regarding the impedance, the real parts show the best agreement among the three specimens. For the imaginary part, the discrepancy becomes larger at higher frequencies. As discussed for specimens Nos. 1 and 2, the measurement and the theory do not agree; however, regarding specimen No. 3, the discrepancies are relatively small compared with the others. This can be attributed to the better manufacturing accuracy in circular hole MPPs-in the case of square holes, not only the sizes but 
also the shape, especially at the corners, was not precise, but in the case of circular holes, the inaccuracy of the shape was smaller and the deviations showed a clear Gaussian distribution, which can be better represented by the average values.

\section{Concluding Remarks}

In this pilot study on the effects of manufacturing accuracy on 3D printed MPPs' acoustical properties, three types of MPPs with square and circular holes were produced by an additive manufacturing 3D printer for consumer use, and their absorption coefficient and acoustic impedances were measured using the impedance tube method. The measured results were discussed in comparison with the theoretical values obtained by Guo's model. From the results, the following conclusions were obtained:

1. In this study, two MPPs with square holes, and one with circular holes were produced and studied. In all cases, the sizes of the holes and the thickness showed deviations. The deviation was larger in the case of square hole MPPs than for circular hole MPPs, in the present study. However, in all cases, the absorption characteristics were similar to those of a typical MPP and showed reasonably high absorption performance. Therefore, it is possible in practice to use the MPPs manufactured by additive manufacturing 3D printers.

2. Although the deviation of the parameters was smaller in the case of circular hole MPPs, the deviation of the parameters made theoretical predictions using averaged parameters difficult. This is because, in the theory, the surface is assumed to be homogeneous; however, in reality, with the deviation of the parameters, the surface is no longer homogeneous.

Therefore, when the parameters of MPPs had a distribution with a large deviation, they did not demonstrate homogeneity, and their acoustical properties were not predictable using the existing theories, which assume a homogeneous surface. In previous studies that dealt with materials with smaller deviations, it was reportedly possible to predict the acoustical properties with the existing theory, e.g., Maa's theory (essentially the same as Guo's model used in this study). However, the samples used in this study appear to have exceeded the range of deviations in which those theories remain applicable. According to the previous studies, hole diameters are the most easily deviated, and the effect is large; thus, it is important to discuss how the deviations in hole diameters should be treated. This perhaps does not need to amend the existing theory itself but introducing statistical treatment may be needed. A method that can be applicable in these cases will be a topic in follow-up studies.

Author Contributions: Conceptualization, K.S. and T.O.; methodology, all authors; validation, all authors; formal analysis, M.K.; investigation, S.N.; data curation, M.K.; Writing-original draft preparation, K.S.; Writing-review and editing, all authors; supervision, K.S., T.O. and S.N.; project administration, K.S. All authors have read and agreed to the published version of the manuscript.

Funding: This research received no external funding.

Conflicts of Interest: The authors declare no conflict of interest.

\section{References}

1. Maa, D.Y. Theory and design of microperforated panel sound absorbing constructions. Scientia Sinica 1975, 18, 55-71.

2. Maa, D.Y. Microperforated-panel wideband absorber. Noise Contr. Eng. J. 1987, 29, 77-84. [CrossRef]

3. Maa, D.Y. Potential of microperforated panel absorber. J. Acoust. Soc. Am. 1998, 104, 2861-2866. [CrossRef]

4. Kang, J.; Fuchs, H.V. Predicting absorption of open weave textiles and micro-perforated membranes backed by an air space. J. Sound. Vib. 1999, 220, 905-920. [CrossRef]

5. Fuchs, H.V.; Zha, X.; Drotleff, H.D. Creating low-noise environments in communication rooms. Appl. Acoust. 2001, 62, 1375-1396. [CrossRef]

6. Zha, X.; Fuchs, H.V.; Drotleff, H.D. Improving the acoustic working conditions for musicians in small spaces. Appl. Acoust. 2002, 63, 203-221. [CrossRef] 
7. Adams, T. Sound Materials: A Compendium of Sound Absorbing Materials for Architecture and Design; Frame Pub.: New York, NY, USA, 2017.

8. Herrin, D.; Liu, J.; Seybert, A. Properties and applications of microperforated panel. Sound Vib. 2011, 45, 6-9.

9. Herrin, D. A guide to the applications of microperforated panel absorbers. Sound Vib. 2017, 51, 12-18.

10. Sakagami, K. Some examples of usage of microperforated panel absorbers. Archit. Acoust. Noise Contr. 2019, 188, 1-7. (In Japanese)

11. Liu, Z.; Zhan, J.; Fard, M.; Davy, J.L. Acoustic properties of multilayer sound absorbers with a 3D printed micro-perforated pane. Appl. Acoust. 2017, 121, 25-32. [CrossRef]

12. Boulvert, J.; Costa-Baptista, J.; Cavalier, T.; Perna, M.; Fotsing, E.R.; Romero-Garcia, V.; Ross, A.; Mardjono, J.; Groby, J.-P. Acoustic modelling of micro-lattice obtained by additive manufacturing. Appl. Acoust. 2020, 164, 107244. [CrossRef]

13. Carbajo, J.; Mosanenzadeh, S.G.; Kim, S.; Fang, N.X. Sound absorption of acoustic resonators with oblique perforations. Appl. Phys. Lett. 2020, 116, 054101. [CrossRef]

14. Nakanishi, S. Sound Absorption of Helmholtz Resonator Included a Winding Built-in Neck Extension; INTER-NOISE and NOISE-CON Congress and Conference Proceedings: Hamburg, Germany, 2016.

15. Pan, L.; Martellotta, F. A parametric study of the acoustic performance of resonant absorbers made of micro-perforated membranes and perforated panels. Appl. Sci. 2020, 10, 1581. [CrossRef]

16. Guo, Y.; Allam, S.; Abom, M. Micro-perforated plates for vehicle applications. In Proceedings of the 2008 Congress and exposition of noise control engineering, Inter-Noise 2008, Shanghai, China, 26-29 October 2008.

17. Bolton, J.S.; Kim, N. Use of CFD to calculate the dynamic resistive end correction for microperforated materials. Acoust. Aust. 2010, 38, 134-144.

18. Herdtle, T.; Bolton, J.S.; Kim, N.; Alexander, J.H.; Gerdes, R.W. Transfer impedance of microperforated materials with tapered holes. J. Acoust. Soc. Am. 2013, 134, 4752-4762. [CrossRef] [PubMed]

19. Okuzono, T.; Nitta, T.; Sakagami, K. Note on microperforated panel model using equivalent-fluid-based absorption elements. Acoust. Sci. Technol. 2019, 40, 221-224. [CrossRef]

20. Cox, T.J.; D'Antonio, P. Acoustic Absorbers and Diffusers: Theory, Design and Application, 3rd ed.; CRC Press: New York, NY, USA, 2017; p. 243.

(C) 2020 by the authors. Licensee MDPI, Basel, Switzerland. This article is an open access article distributed under the terms and conditions of the Creative Commons Attribution (CC BY) license (http://creativecommons.org/licenses/by/4.0/). 